\title{
EDITORIAL
}

\section{Neuroimmunotherapeutics Comes of Age}

This issue of Neurotherapeutics addresses immune therapies for neurological disease. Much of the issue is focused on multiple sclerosis (MS), which is the most important immunologically-mediated neurological disorder from the standpoint of number affected and burden of morbidity. Furthermore, MS has been the object of increasingly intense research scrutiny during recent decades. However, an attempt has been made to expand the field of view to encompass other disorders, often bringing to bear insights into neuroimmunology and therapeutic intervention in neuroinflammation, derived from MS research.

We have been fortunate to recruit authentic expert clinician/scientists as contributors to this issue. Each contribution was prepared by a leading investigator with an active research program in the topic of discussion. This issue is organized into three sections, which is described as follows.

In the first section, we assembled current reviews of some of the major players in the inflammatory processes of the nervous system, while maintaining an emphasis on the therapeutic implications of such knowledge. Monica Carson and colleagues wrote about microglia (pages 571-579), the intrinsic inflammatory and immune cells of the CNS. V. Wee Yong and coworkers discussed the matrix metalloproteinases (pages 580-589). In addition, Carine Savarin-Vuaillat and Richard Ransohoff described the chemokine and chemokine receptor system (pages 590-601). All together, these reviews address the intrinsic inflammatory and immune cells of the CNS (microglia), an important effector and modulatory system (MMPs), and the apparatus for recruiting and activating leukocytes from the bloodstream (chemokines). Rohit Bakshi and colleagues (pages 602-617) provided an indepth discussion of magnetic resonance imaging, which constitutes a critical means for applying any therapeutic initiatives for MS.

In the second section, current therapies for MS are discussed. Elliot Frohman and coworkers (pages 618626 and 627-632) discussed the application of corticosteroids, a drug class often used, but too little discussed. Frohman and coworkers addressed both the use of these agents in MS attacks and the novel concept that diseasemodifying effects can be mediated by corticosteroids in a fashion that maintains acceptable risk-benefit ratios. Richard Rudick and Rob Bermel (pages 633-646) com- prehensively reviewed interferon-beta treatment for MS, tackling the most widely used, yet still poorly understood agent for this disease. Martin Weber, Reinhard Hohlfeld, and Scott Zamvil discussed glatiramer acetate, considering its decades-long history and describing research as recently published as Summer, 2007 (pages 647-653). Peter Hartung and colleagues (pages 654-660) provided an overview of the immunosuppressants that form the mainstay of second-stage therapy for the large fraction of our patients for whom the first-line agents do not adequately control the disease.

The third section looks toward the future. This section begins with Larry Steinman's essay on the desired endpoint of all MS research (pages 661-665) (i.e., a therapy that eliminates the pathogenic autoreactivity without immunosuppression). Samia Khoury and Vissia Viglietta (pages 666-675) described how the requirement of the $\mathrm{T}$ cell for co-stimulation by the antigen-presenting cell at the time of antigen recognition might be used to modify or inhibit pathogenic autoimmunity in MS. Paolo Muraro and Bibi Bielekova gave a wide range and scholarly series of comments regarding varied new approaches to MS therapy (pages 676-692), all of which could potentially reach full-scale clinical application; however, not all of these approaches will be achieved to reach this endpoint. Martin Weber, Larry Steinman, and Scott Zamvil gave an in-depth treatment of the means by which the statins (i.e., drugs that have changed the face of atherosclerosis management) may also impact inflammatory and neurodegenerative disease (pages 693-700). The current volume concludes with reviews concerning two highly exciting topics. Jaime Imitola instructs us on the prospects for the use of stem cells or progenitor cells for therapeutics (pages 701-714), which includes a full recognition of the need to understand both the disease under consideration and the biology of the cell being applied for its amelioration. Pedro Lowenstein, Kurt Kroger, and Maria Castro conclude this issue of the journal with their presentation of the remarkable promise of gene therapy using viral vectors (pages 715-724), and they remind us that the immune and inflammatory reaction of the host to such interventions must be acknowledged and understood.

Throughout, we have tried to provide important information that we believe to be authoritative, timely, 
and practical, if only in the sense of responding to patient questions about what is on the horizon. The volume is not comprehensive, due to space limitations. What is missing? We were not able to include the peripheral nervous system immune/inflammatory disorders or their treatments such as intravenous immunoglobulin. We did not discuss plasma exchange as a treatment for CNS and peripheral nervous system disorders. We regret these and other omissions occasioned by the space available for this volume. It is our hope that the material presented here will be helpful for the readers of this journal, and that it gives a full measure of recognition both for the distance traveled in the conduct of this research and for the miles left to go.
Richard M. Ransohoff, MD

Department of Neurosciences Neuroinflammation Research Center

Lerner Research Institute and Mellen Center for MS Treatment and Research

Cleveland Clinic Foundation

9500 Euclid Avenue

Cleveland, OH 44195

Scott S. Zamvil, MD, PhD

Department of Neurology and Program in Immunology University of California, San Francisco

513 Parnassus Avenue

Rm. S-268

San Francisco, CA 94143 